\title{
A LIMING PROCESS WITH SODIUM SILICATE COMPOUND AS A SWELLING AGENT
}

\section{PROCES DE CENUŞĂRIRE CU UN COMPUS PE BAZĂ DE SILICAT DE SODIU CA AGENT DE GONFLARE}

\author{
Xiaoyun JIAN ${ }^{1}$, Bo TENG ${ }^{1}$, Jinwei ZHANG ${ }^{1}$, Yanping GAO ${ }^{1}$, Wuyong $\mathrm{CHEN}^{1,2^{*}}$ \\ ${ }^{1}$ National Engineering Laboratory for Clean Technology of Leather Manufacture, 610065, Chengdu, China \\ ${ }^{2}$ Key Laboratory for Leather Chemistry and Engineering of the Education Ministry, 610065, Chengdu, China
}

\begin{abstract}
A LIMING PROCESS WITH SODIUM SILICATE COMPOUND AS A SWELLING AGENT
ABSTRACT. Low modulus sodium silicate was prepared with a polysilicon byproduct-silicon tetrachloride, and used as a swelling agent. Calcium chloride, hydrazine and protease were selected as auxiliaries for the swelling agent. Dosage of the auxiliaries was optimized through the weight increase, thickness increase, proteoglycan removing and skin histology in swelling. Based on the auxiliary optimization, a sodium silicate compound with $67 \%$ sodium silicate, $17 \%$ hydrazine and $3 \%$ protease (SSC swelling agent) was prepared as a swelling agent and its swelling ability was investigated with SEM and proteoglycan removing analysis. Also, the environmental impact of the swelling process was evaluated by total solid of the effluent. The results showed that a comparable opening up of fiber bundles was obtained with $6 \%$ SSC swelling agent (based on the unhaired weight) and proteoglycan removal in the skin was improved. Compared with a traditional liming process, there was a less total solid in the effluents. The results could provide a new valuable reference for cleaner swelling process.

KEY WORDS: leather making, liming, swelling, sodium silicate, polysilicon
\end{abstract}

PROCES DE CENUSARIRE CU UN COMPUS PE BAZA DE SILICAT DE SODIU CA AGENT DE GONFLARE

REZUMAT. S-a preparat un silicat de sodiu cu modul mic utilizând un produs secundar al siliciului policristalin - tetraclorura de siliciu - si s-a folosit ca agent de gonflare. Clorura de calciu, hidrazina si proteaza au fost selectate ca auxiliari pentru agentul de gonflare. Dozarea auxiliarilor a fost optimizata determinând cresterea în greutate, cresterea în grosime, gradul de îndepartare a proteoglicanilor si histologia pielii la gonflare. Pe baza optimizarii auxiliarilor, s-a preparat un compus de silicat de sodiu cu continut de $67 \%$ silicat de sodiu, $17 \%$ hidrazina si $3 \%$ proteaza (agent de gonflare SSC) ca agent de gonflare, iar capacitatea acestuia de gonflare a fost investigata prin microscopia SEM si analiza gradului de îndepartare a proteoglicanilor. S-a evaluat, de asemenea, impactul asupra mediului al procesului de gonflare prin determinarea continutului de materii solide totale al efluentului. Rezultatele au aratat ca s-a obtinut o deschidere a fasciculelor de fibre comparabila utilizând agentul de gonflare SSC în concentratie de 6\% (raportat la greutatea pielii fara par), iar îndepartarea proteoglicanilor din piele a fost îmbunatatita. Comparativ cu un proces traditional de cenusarire, a existat un continut mai mic de materii solide totale în efluenti. Rezultatele ar putea oferi o noua referinta valoroasa pentru un proces de gonflare mai curat.

CUVINTE CHEIE: fabricarea pielii, var, gonflare, silicat de sodiu, siliciu policristalin

\section{UN PROCESSUS DE CHAULAGE EN UTILISANT UN COMPOSÉ DU SILICATE DE SODIUM COMME AGENT GONFLANT}

RÉSUMÉ. Le silicate de sodium $r$ faible module a été préparé en utilisant un sous-produit de silicium polycristallin - le tétrachlorure de silicium - et utilisé en tant qu'agent de gonflement. Le chlorure de calcium, l'hydrazine et la protéase ont été sélectionnés en tant qu'auxiliaires pour l'agent gonflant. Le dosage des auxiliaires a été optimisé par l'augmentation de poids, l'augmentation de l'épaisseur, l'enlcvement des protéoglycanes et l'histologie de la peau gonflée. R base de I'optimisation des auxiliaires, on a préparé un composé de silicate de sodium avec $67 \%$ silicate de sodium, $17 \%$ hydrazine et $3 \%$ protease (agent gonflant SSC) comme agent de gonflement et sa capacité de gonflement a été étudiée par la microscopie SEM et l'analyse d'enlcvement des protéoglycanes. En outre, l'impact environnemental du processus de gonflement a été évalué par la tenure en maticres solides totales de l'effluent. Les résultats ont montré qu'une ouverture correspondante des faisceaux de fibres a été obtenue avec $6 \%$ agent de gonflement SSC (par rapport au poids du peau épilée) et l'enlcvement des protéoglycanes dans la peau est amélioré. Par rapport r un procédé traditionnel de chaulage, il y avait moins solides totales dans les effluents. Les résultats pourraient fournir une nouvelle référence précieuse pour un processus de gonflement plus propre.

MOTS CLÉS: fabrication du cuir, chaulage, gonflement, silicate de sodium, silicium polycristallin

\section{INTRODUCTION}

The fiber opening process determines the penetration of chemicals in leather making, also affects the sensory and physical properties of leather. However, some disadvantages of traditional liming are always mentioned by leather chemists, including lower solubility and sludge in liming effluent [1-3].

\section{INTRODUCERE}

Procesul de deschidere a fibrelor determină pătrunderea substanţelor chimice în piele, afectând, de asemenea, proprietăţile senzoriale şi fizice ale acesteia. $\mathrm{Cu}$ toate acestea, chimiştii pielari menţionează întotdeauna unele dezavantaje ale procesului tradiţional de cenuşărire, care includ solubilitatea mai mică şi prezenţa nămolului în efluentul de la cenuşărire [1-3].

* Correspondence to: Wuyong CHEN, National Engineering Laboratory for Clean Technology of Leather Manufacture, Key Laboratory for Leather Chemistry and Engineering of the Education Ministry, 610065, Chengdu, China, wuyong.chen@163.com 
To overcome the disadvantages in a traditional liming process, many kinds of lime-free fiber opening agents were discovered [4-5]. One of the lime-free swelling agents was sodium silicate. It had been proved that the fiber opening ability of sodium silicate was comparable to that of a conventional process [6]. Meanwhile, sodium silicate will not have any negative impact for leather. Compared with a traditional liming process, the new process has a lower $\mathrm{COD}, \mathrm{BOD}_{5}$ and total solid (TS) data in swelling effluent [7-8]. In this study, a polysilicon by-product silicon tetrachloride was used as a raw material for preparing a silicate compound with hydrazine and protease to get a moderate swelling. In the process with the compound swelling agent, the fiber opening ability of skin was studied and the environmental impact was evaluated with TS. The results could provide a valuable reference for both polysilicon and leather industry.

\section{EXPERIMENT}

\section{Materials}

Wet salted goatskins were selected as a raw material. The sodium chloride content and humidity of goatskins were respectively $25 \% \sim 30 \%$ and $35 \% \sim 40 \%$. All the chemicals used in leather making process were commercial grade. Silicon tetrachloride was a polysilicon byproduct collected from Yongxiang polysilicon Limited Liability Company in China. The alkaline protease (2709 protease) was purchased from Long Kete Limited Liability Company in China. Chemicals used for the analysis were all research grade.

\section{Preparation of Sodium Silicate}

$100 \mathrm{~g}$ silicon tetrachloride was added into $300 \mathrm{~g}$ ice water $\left(T \leq 4^{\circ} \mathrm{C}\right)$, and then heated at $120^{\circ} \mathrm{C}$ to remove the hydrochloric acid. A $10 \mathrm{~mL}$ water and equal weight sodium hydroxide were added to get sodium silicate solution. The properties of this sodium silicate solution were analyzed according to GB/T 4209-2008 [9]. The results showed the content of $\mathrm{Na}_{2} \mathrm{O}$ and $\mathrm{SiO}_{2}$ in the sodium silicate
Pentru a depăşi dezavantajele unui proces tradiţional de cenuşărire, au fost descoperite multe tipuri de agenţi fără conţinut de var pentru deschiderea fibrelor [4-5]. Unul dintre aceşti agenţi de gonflare fără var a fost silicatul de sodiu. S-a demonstrat că silicatul de sodiu are o capacitate de deschidere a fibrelor comparabilă cu cea obţinută printr-un proces convenţional [6]. În acelaşi timp, silicatul de sodiu nu are niciun impact negativ asupra pielii. Comparativ cu un proces tradiţional de cenuşărire, noul proces are valori mai mici pentru $\mathrm{CCO}, \mathrm{CBO}_{5}$ şi materii solide totale (TS) în apele reziduale de la gonflare [7-8]. În acest studiu s-a utilizat ca materie primă un produs secundar al siliciului policristalin, tetraclorura de siliciu, pentru prepararea unui compus silicat cu hidrazină şi protează în vederea obţinerii unui grad de gonflare moderat. În procesul în care s-a utilizat agentul de gonflare, s-a studiat capacitatea acestuia de deschidere a fibrelor pielii şi s-a evaluat impactul asupra mediului prin intermediul conţinutului de TS. Rezultatele ar putea oferi o referinţă valoroasă atât pentru industria siliciului policristalin, cât şi pentru industria de pielărie.

\section{PARTEA EXPERIMENTALĂ}

\section{Materiale}

Ca materie primă s-au selectat piei caprine umed sărate. Conţinutul de clorură de sodiu şi umiditatea pieilor caprine au fost de $25 \% \sim 30 \%$, respectiv 35\% 40\%. Toate substanţele chimice utilizate în procesul de fabricare a pielii au fost de calitate comercială. Tetraclorura de siliciu a fost un produs secundar al siliciului policristalin furnizat de compania Yongxiang din China. Proteaza alcalină (protează 2709) s-a achiziţionat de la compania Long Kete din China. Toate substanţele chimice utilizate pentru analiză au fost de calitate analitică.

\section{Prepararea silicatului de sodiu}

S-au adăugat $100 \mathrm{~g}$ tetraclorură de siliciu în $300 \mathrm{~g}$ apă cu gheaţă $\left(T \leq 4^{\circ} \mathrm{C}\right)$, apoi s-a încălzit la $120^{\circ} \mathrm{C}$ pentru a elimina acidul clorhidric. S-au adăugat $10 \mathrm{ml}$ apă şi hidroxid de sodiu în greutate egală pentru a obţine o soluţie de silicat de sodiu. Proprietăţile acestei soluţii de silicat de sodiu au fost analizate conform standardului GB/T 4209-2008 [9]. Rezultatele au arătat că soluţia de silicat de sodiu a conţinut $\mathrm{Na}_{2} \mathrm{O}$ şi $\mathrm{SiO}_{2}$ în 
solution were $12.92 \%$ and $15.87 \%$, respectively, modulus was 1.27 , total dissolved solid was $28.76 \%$ and $\mathrm{pH}$ was 13.50 .

\section{Optimization of Auxiliaries for Compound Swelling Agent}

Goat skins were soaked as usual, and the soaked skins were unhaired with a neutral protease (1398 protease) according to conventional dip and pile method. The unhaired pelts were cut into pieces along the backbones. The left sides were used for the following trials. $4 \%$ sodium silicate, $\mathrm{X} \%$ auxiliary, and $300 \%$ water (based on the weight of unhaired pelt) were added into the drum $(2600 \times 950 \times 1450 \mathrm{~mm})$ for swelling. The drums were run for $5 \mathrm{~min}$ per hour in 7 hours then standing overnight. The right sides were prepared with a conventional liming process as a control.

The optimized sodium silicate compound with $67 \%$ sodium silicate, $17 \%$ hydrazine and $3 \%$ protease (SSC swelling agent) was prepared for liming as above process.

\section{Weight and Thickness of Swelled Pelt}

The weight increase was calculated as follows:

$$
\underset{\text { Weight increase }}{\text { Creşterea greutăţii }}=\frac{w_{2}-w_{1}}{w_{1}} \times 100 \%
$$

where $w_{1}$ - weight of unhaired pelt, $w_{2}$ - weight of swelled pelt.

The thickness increase was calculated as follows:

$$
\begin{aligned}
& \text { Thickness increase } \\
& \text { Creşterea grosimii }
\end{aligned}
$$

where $d_{1}$ - thickness of unhaired pelt, $d_{2}$ - thickness of swelled pelt.

\section{Histology of Swelled Skin}

The skins prepared with swelling agent were cut from the official sampling position. The samples were fixed with neutral formaldehyde over $24 \mathrm{~h}$ and sliced proporţie $12,92 \%$, respectiv $15,87 \%$; modulul a fost de 1,27 , conţinutul de materii solide totale dizolvate a fost de $28,76 \%$ şi pH-ul a fost 13,50 .

\section{Optimizarea auxiliarilor pentru agentul de gonflare}

Pieile de capră au fost înmuiate printr-un proces uzual, apoi s-a îndepărtat părul de pe pieile înmuiate utilizând o protează neutră (protează 1398) conform metodei convenţionale. Pieile gelatină fără păr au fost tăiate în bucăţi de-a lungul şirei spinării. Bucăţile din partea stângă au fost utilizate pentru experimente. Sau adăugat în butoi $(2600 \times 950 \times 1450 \mathrm{~mm}) 4 \%$ silicat de sodiu, X\% auxiliar şi 300\% apă (raportat la greutatea pielii gelatină fără păr) pentru gonflare. Butoiul a funcţionat 5 minute pe oră timp de 7 ore, apoi a fost lăsat să se odihnească peste noapte. Bucăţile din partea dreaptă au fost prelucrate printr-un procedeu convenţional cu var şi luate ca probe de referinţă.

Compusul pe bază de silicat de sodiu optimizat cu $67 \%$ silicat de sodiu, $17 \%$ hidrazină şi $3 \%$ protează (agent de gonflare SSC) a fost preparat pentru cenuşărire conform procesului de mai sus.

\section{Greutatea şi grosimea pielii gelatină gonflate}

Creşterea greutăţii a fost calculată după cum urmează:

unde w $_{1}$ - greutatea pielii gelatină fără păr, $w_{2}$ greutatea pielii gelatină gonflate.

Creşterea în grosime a fost calculată după cum urmează:

$$
=\frac{d_{2}-d_{1}}{d_{1}} \times 100 \%
$$

unde $d_{1}$ - grosimea pielii gelatină fără păr, $d_{2}$ - grosimea pielii gelatină gonflate.

\section{Histologia pielii gonflate}

Pieile prelucrate cu agent de gonflare au fost tăiate conform zonelor standard de eşantionare. Probele au fost fixate cu formaldehidă neutră timp de 24 de ore şi 
into pieces $(13 \mu \mathrm{m})$ with freezing microtome. The samples were then immobilized with protein fluid and stained according to hematoxylin-eosin method. A SZX12 optical microscope (OLYMPUS Company) was used for the observation.

\section{Liming with SSC Swelling Agent}

The unhaired skins were cut into sides along the backbones. The left sides were taken for experimental and right sides for control. The experimental process (see Optimization of auxiliaries for compound swelling agent) was carried out with SSC swelling agent $(6 \%)$. The control swelling was performed according to a conventional liming process.

\section{Scanning Electron Microscope Analysis}

The unhaired pelt was cut into two sides along the backbone. One side was limed with SSC swelling agent as a sample. The other side was prepared with a traditional liming process as a control. The samples were then dehydrated with ethyl alcohol and observed with a JSM-5900 scanning electron microscope (Philips Company).

\section{Analysis of Proteoglycans in Waste Liquors}

$100 \mathrm{~mL}$ swelling effluent was filtrated and then analyzed for proteoglycan by the method of Mantle, $\mathrm{M}$. etal. [10].

\section{Analysis of Total Solid in Waste Liquors}

$100 \mathrm{~mL}$ swelled effluent was collected and analyzed for total solid (TS) following the procedure of Albu, L. et al. [11]. tăiate în bucăţi $(13 \mu \mathrm{m})$ cu un microtom cu congelare. Probele au fost apoi imobilizate cu lichid de proteine şi s-au colorat cu hematoxilină-eozină. Pentru observare s-a utilizat un microscop optic SZX12 (firma OLYMPUS).

\section{Cenuşărirea cu agentul de gonflare SSC}

Pieile depărate au fost tăiate în bucăţi de-a lungul şirei spinării. Bucăţile din partea stângă au fost folosite pentru experimente, iar cele din partea dreaptă au fost luate ca probe de referinţă. Procesul experimental (a se vedea Optimizarea auxiliarilor pentru agentul de gonflare) a fost realizat utilizând agentul de gonflare SSC (6\%). Gonflarea probelor martor s-a realizat conform unui procedeu convenţional de cenuşărire.

\section{Analiza prin microscopie electronică de baleiaj}

Pieile depărate au fost tăiate în două părţi de-a lungul şirei spinării. O parte a fost cenuşărită cu agent de gonflare SSC ca probă de testare. Cealaltă parte a fost prelucrată utilizând un procedeu tradiţional cu var ca probă martor. Probele au fost apoi deshidratate cu alcool etilic şi observate cu un microscop electronic de baleiaj JSM-5900 (firma Philips).

\section{Determinarea conţinutului de proteoglicani din efluenţi}

S-au filtrat $100 \mathrm{ml}$ din efluentul de la gonflare şi apoi s-au analizat pentru determinarea proteoglicanilor prin metoda propusă de Mantle, M. şi colab. [10].

\section{Determinarea conţinutului de solide totale din efluenţi}

S-au colectat $100 \mathrm{ml}$ de efluent de la gonflare şi sau analizat pentru determinarea solidelor totale (TS) urmând procedura descrisă de Albu, L. şi colab. [11]. 


\section{RESULTS AND DISCUSSION}

\section{Optimization of Auxiliaries for Compound Swelling Agent}

\section{REZULTATE ŞI DISCUTII}

\section{Optimizarea auxiliarilor pentru agentul de gonflare}

Table 1: Effect of auxiliaries on the proteoglycan removal

Tabelul 1: Influenţa auxiliarilor asupra îndepărtării proteoglicanilor

\begin{tabular}{|c|c|c|c|c|c|}
\hline $\begin{array}{l}\text { Sample } \\
\text { Proba }\end{array}$ & $\begin{array}{l}\text { Sodium silicate- } \mathrm{CaCl}_{2} \\
\text { Silicat de sodiu- } \mathrm{CaCl}_{2}\end{array}$ & $\begin{array}{l}\text { Sodium silicate- } \\
\text { hydrazine } \\
\text { Silicat de sodiu- } \\
\text { hidrazină }\end{array}$ & $\begin{array}{l}\text { Sodium silicate- } 2709 \\
\text { protease } \\
\text { Silicat de sodiu- } \\
\text { protează } 2709\end{array}$ & $\begin{array}{l}\text { Sodium silicate } \\
\text { Silicat de sodiu }\end{array}$ & $\begin{array}{l}\text { Control (lime) } \\
\text { Martor (var) }\end{array}$ \\
\hline $\begin{array}{c}\text { Proteoglycan removal / (mg/L) } \\
\text { Gradul de îndepărtare a } \\
\text { proteoglicanilor / (mg/l) }\end{array}$ & 41.67 & 52.59 & 55.17 & 39.12 & 32.68 \\
\hline
\end{tabular}

The effect of liming could be characterized with the proteoglycan removal in liming liquors. The effect of auxiliaries on the proteoglycan removal was shown in Table 1. It indicated that all of the silicate with $\mathrm{CaCl}_{2}$, hydrazine and protease presented a better proteoglycan removal ability, compared with a traditional liming process. Among them, hydrazine and protease showed a significant effects, increase of proteoglycan removal was $52.59 \%$ and $55.17 \%$, respectively. So, the hydrazine and protease were selected as auxiliaries of SSC swelling agent.

\section{Optimization of the Dosage of Hydrazine}

Efectul cenuşăririi poate fi caracterizat prin eliminarea proteoglicanilor din soluţia de cenuşărire. Influenţa auxiliarilor asupra eliminării proteoglicanilor este prezentată în Tabelul 1. Acesta arată că toţi silicaţii cu conţinut de $\mathrm{CaCl}_{2}$, hidrazină şi protează au prezentat o capacitate mai bună de îndepărtare a proteoglicanilor, comparativ cu un proces tradiţional de cenuşărire. Dintre aceştia, cei cu hidrazină şi protează au prezentat un efect semnificativ, gradul de îndepărtare a proteoglicanilor crescând până la 52,59\%, respectiv 55,17\%. Aşadar, hidrazina şi proteaza au fost selectate ca auxiliari ai agentului de gonflare SSC.

\section{Optimizarea dozei de hidrazină}

Table 2: Effect of hydrazine dosage on the swelling ability Tabelul 2: Influenţa dozei de hidrazină asupra capacităţii de gonflare

\begin{tabular}{|c|c|c|c|}
\hline $\begin{array}{c}\text { Dosage of } \\
\text { hydrazine* / (\%) } \\
\text { Doza de } \\
\text { hidrazină } \breve{~}^{*}(\%)\end{array}$ & $\begin{array}{c}\text { Weight } \\
\text { increase / (\%) } \\
\text { Creşterea în } \\
\text { greutate / (\%) }\end{array}$ & $\begin{array}{l}\text { Thickness } \\
\text { increase / (\%) } \\
\text { Creşterea în } \\
\text { grosime / (\%) }\end{array}$ & $\begin{array}{l}\text { Proteoglycan removal / } \\
\text { (mg/L) } \\
\text { Gradul de îndepărtarea } \\
\text { proteoglicanilor / (mg/l) }\end{array}$ \\
\hline 0.5 & 58.90 & 50.44 & 42.34 \\
\hline 1.0 & 70.26 & 76.4 & 56.66 \\
\hline 1.5 & 60.11 & 60.75 & 52.39 \\
\hline 2.0 & 64.34 & 50.58 & 51.62 \\
\hline
\end{tabular}




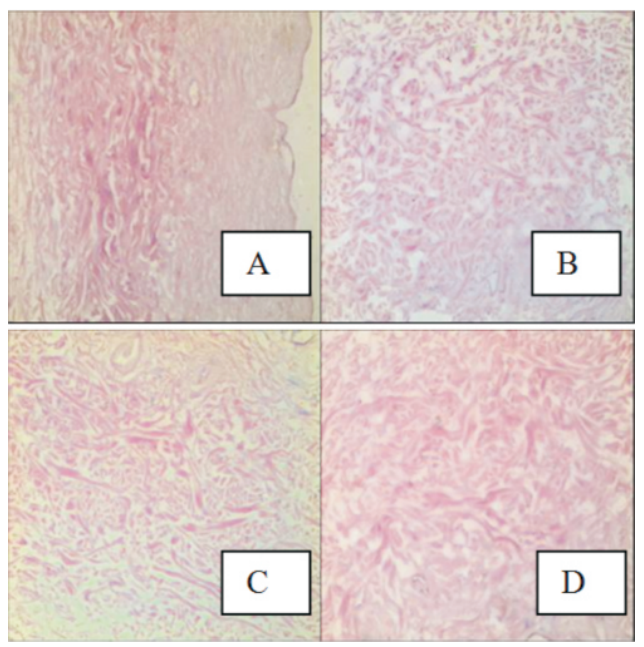

Figure 1. Histological micrographs of samples after fiber opening with sodium silicate and different dosages of hydrazine $(\times 40)$ : A - $0.5 \%$ hydrazine, B $-1.0 \%$ hydrazine, C - $1.5 \%$ hydrazine, D - $2.0 \%$ hydrazine

Figura 1. Micrografiile histologice ale probelor după deschiderea fibrelor cu silicat de sodiu şi diferite doze de hidrazină (x40): A - 0,5\% hidrazină, B - 1,0\% hidrazină, C - 1,5\% hidrazină, D - 2,0\% hidrazină

The weight increase and thickness increase, the same as proteoglycan removal, could characterize the effect of skin swelling. Table 2 showed that they were correlated with the dosage of hydrazine. Swelling ability was raised with the dosage and reached to the highest at $1.0 \%$. At the level, the weight increase and thickness increase and proteoglycan removal reached $70.26 \%, 76.4 \%$ and $56.66 \%$, respectively. Also $1.0 \%$ hydrazine dosage showed a better fiber opening (Figure 1B). However, the higher dosage of hydrazine might result in a poorer effect of fiber opening (Figure $1 C, D)$. Therefore, $1.0 \%$ of hydrazine was used as auxiliary of SSC swelling agent.

\section{Optimization of the Dosage of Protease}

Similar îndepărtării proteoglicanilor, creşterea în greutate şi creşterea în grosime pot caracteriza efectul de gonflare a pielii. Tabelul 2 arată că aceste creşteri au fost corelate cu doza de hidrazină. Capacitatea de gonflare a crescut odată cu doza şi a atins maximum la concentraţia de 1,0\%. La acest nivel, creşterea în greutate, creşterea în grosime şi eliminarea proteoglicanilor au ajuns la $70,26 \%$, $76,4 \%$, respectiv $56,66 \%$. De asemenea, doza de $1,0 \%$ hidrazină a dus la o mai bună deschidere a fibrelor (Figura 1B). Cu toate acestea, o doză mai mare de hidrazină ar putea conduce la un efect mai slab de deschidere a fibrelor (Figura 1C, D). Prin urmare, hidrazina în proporţie de 1,0\% a fost utilizată ca auxiliar pentru agentul de gonflare SSC.

Table 3: Effect of protease dosage on the swelling ability Tabelul 3: Influenţa dozei de protează asupra capacităţii de gonflare

\begin{tabular}{|c|c|c|c|}
\hline $\begin{array}{l}\text { Dosage of } \\
\text { protease* / (\%) } \\
\text { Doza de } \\
\text { protează* /(\%) }\end{array}$ & $\begin{array}{l}\text { Weight increase } \\
\text { / (\%) } \\
\text { Creşterea în } \\
\text { greutate / (\%) }\end{array}$ & $\begin{array}{l}\text { Thickness } \\
\text { increase / (\%) } \\
\text { Creşterea în } \\
\text { grosime / (\%) }\end{array}$ & $\begin{array}{l}\text { Proteoglycan removal / } \\
\text { (mg/L) } \\
\text { Gradul de îndepărtare a } \\
\text { proteoglicanilor / (mg/l) }\end{array}$ \\
\hline 0.10 & 56.33 & 80.74 & 49.85 \\
\hline 0.15 & 67.86 & 78.11 & 31.59 \\
\hline 0.20 & 70.42 & 93.33 & 60.33 \\
\hline 0.25 & 46.15 & 69.2 & 49.23 \\
\hline
\end{tabular}




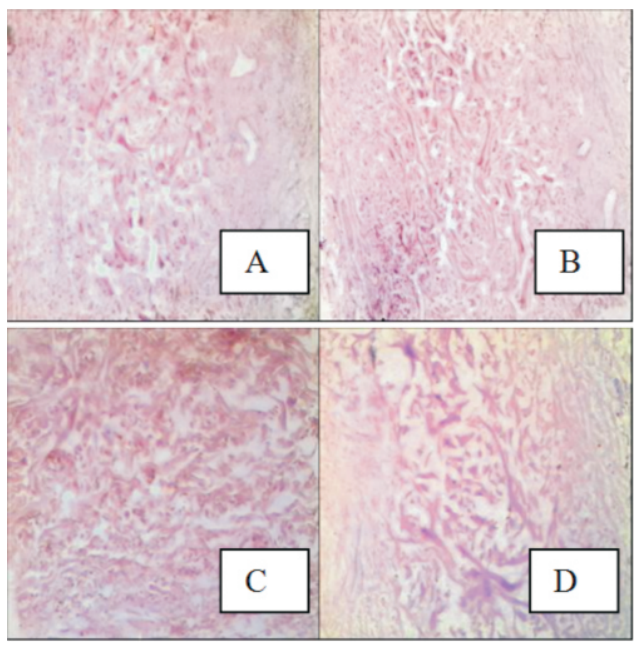

Figure 2. Histological micrographs of samples after fiber opening with sodium silicate and different dosages of protease (×40): A - $0.1 \%$ alkali protease, B - $0.15 \%$ alkali protease,

C - $0.20 \%$ alkali protease, D - $0.25 \%$ alkali protease

Figura 2. Micrografiile histologice ale probelor după deschiderea fibrelor cu silicat de sodiu şi diferite doze de protează (x40): A - 0,1\% protează alcalină, B - 0,15\% protează alcalină,

C - $0,20 \%$ protează alcalină, D - $0,25 \%$ protează alcalină

Table 3 indicated the swelling ability of the compound swelling agent was affected with content of the protease. The weight increase, thickness increase and proteoglycan removal of skin were highest at $0.20 \%$ protease level, reached $70.42 \%, 93.33 \%$ and $60.33 \%$, respectively. Also, histological micrographs showed that $0.20 \%$ protease was sufficient for fiber opening (Figure 2C). Moreover, partial fiber broken was observed when the dosage of protease rose to $0.25 \%$ (Figure 2D). Therefore, $0.20 \%$ of protease was selected as auxiliary of SSC swelling.

Evaluation of Fiber Opening with SSC Swelling Agent
Conform Tabelului 3, capacitatea de gonflare a agentului de gonflare a fost afectată de conţinutul de protează. Creşterea greutăţii, creşterea grosimii şi gradul de îndepărtare a proteoglicanilor din piele au fost mai ridicate la concentraţia de protează de 0,20\%, ajungând la 70,42\%, $93,33 \%$, respectiv $60,33 \%$. De asemenea, micrografiile histologice au arătat că o cantitate de $0,20 \%$ protează a fost suficientă pentru deschiderea fibrelor (Figura 2C). Mai mult decât atât, s-a observat o rupere parţială a fibrelor atunci când doza de protează a crescut la 0,25\% (Figura 2D). Prin urmare, s-a selectat concentraţia de $0,20 \%$ protează ca auxiliar pentruagentul degonflareSSC.

\section{Evaluarea gradului de deschidere a fibrelor $\mathrm{cu}$ agentul de gonflare SSC}

Table 4: Proteoglycan removal for pelts

Tabelul 4: Îndepărtarea proteoglicanilor din pielea gelatină

\begin{tabular}{c|c|c} 
Sample & $\begin{array}{c}\text { Lime } \\
\text { Proba }\end{array}$ & $\begin{array}{c}\text { SSC swelling agent } \\
\text { Agent de gonflare SSC }\end{array}$ \\
\hline $\begin{array}{c}\text { Proteoglycan removal / }(\mathrm{mg} / \mathrm{L}) \\
\text { Gradul de îndepărtare } a \\
\text { proteoglicanilor } /(\mathrm{mg} / \mathrm{l})\end{array}$ & 33.29 & 57.44 \\
\hline
\end{tabular}



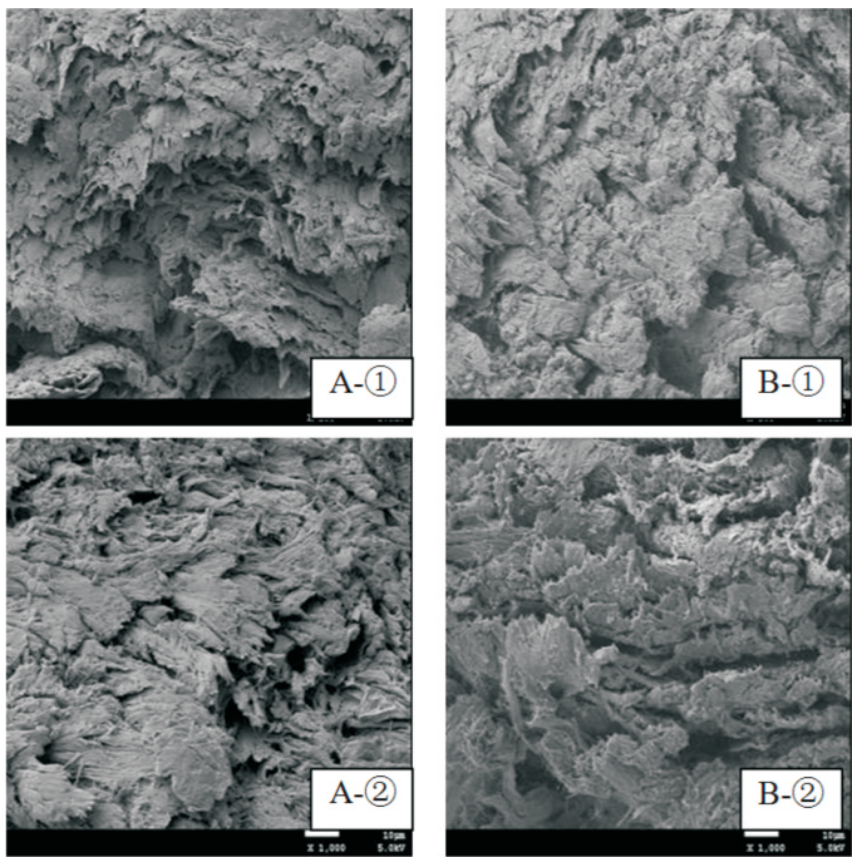

Figure 3. Scanning electron micrographs (SEM) of swelled pelts (A: experimental, B: control; (1): x500, (2): x1000)

Figura 3. Micrografii electronice (SEM) ale pieilor gelatină gonflate

(A: probă experimentală, B: martor; (1): x500, (2): x1000)

After optimization, a sodium silicate compound with $67 \%$ sodium silicate, $17 \%$ hydrazine and $3 \%$ protease (SSC swelling agent) was prepared and used for liming process. The liming process was performed with 6\% SSC swelling agent (based on the unhaired weight). The control swelling was carried out according to a conventional liming process. Proteoglycan removal of skin with SSC swelling agent was shown in Table 4. The SSC swelling agent resulted in much more proteoglycan removal $(57.44 \mathrm{mg} / \mathrm{L})$, compared with the control (33.29 mg/L), showing a better liming effect with the swelling agent. Also, SEM images showed a comparable void spaces in fiber bundles for the experimental and control (see Figure $3 A, B)$, indicating the same opening up of fiber bundles with the SSC swelling agent as the usual liming process.
După optimizare, s-a preparat un compus pe bază de silicat de sodiu cu conţinut de $67 \%$ silicat de sodiu, $17 \%$ hidrazină şi 3\% protează (agent de gonflare SSC) şi s-a utilizat în procesul de cenuşărire. Acesta a fost efectuat utilizând 6\% agent de gonflare SSC (în raport cu greutatea pielii fără păr). Gonflarea probei martor sa realizat conform unui proces convenţional de cenuşărire. Gradul de îndepărtare a proteoglicanilor din piele cu agentul de gonflare SSC a fost prezentat în Tabelul 4. Agentul de gonflare SSC a dus la îndepărtarea unei cantităţi mai mari de proteoglicani $(57,44 \mathrm{mg} / \mathrm{l})$, comparativ cu martorul $(33,29 \mathrm{mg} / \mathrm{l})$, utilizarea agentului de gonflare prezentând un efect de cenuşărire mai bun. De asemenea, imaginile SEM au arătat că spaţiile goale dintre fasciculele de fibre sunt comparabile (vezi Figura 3A, B) pentru probe şi martor, indicând aceeaşi deschidere a fasciculelor de fibre cu agentul de gonflare SSC ca în cazul utilizării procesului de cenuşărire convenţional. 


\section{Impactul asupra mediului la utilizarea agentului de gonflare SSC}

Table 5: TS in waste liquor

Tabelul 5: Conţinutul de materii solide totale al efluentului

\begin{tabular}{c|c|c}
\hline $\begin{array}{c}\text { Sample } \\
\text { Proba }\end{array}$ & $\begin{array}{c}\text { Lime } \\
\text { Var }\end{array}$ & $\begin{array}{c}\text { SSC swelling agent } \\
\text { Agent de gonflare SSC }\end{array}$ \\
\hline $\begin{array}{c}\text { TS / (g/100mL) } \\
\text { Materii solide totale / } \\
(\mathrm{g} / 100 \mathrm{ml})\end{array}$ & 1.5763 & 1.0547 \\
\hline
\end{tabular}

As shown in Table 5, the TS of the experimental was lower $(1.0547 \mathrm{~g} / 100 \mathrm{~mL})$ and decreased by $33 \%$ compared with the control. Usually, lime is a major contributor for TS in a waste liquor of liming process due to its lower solubility. Since there was no lime in SSC swelling agent, TS from lime was eliminated in the waste liquor. Only some hair and small pieces of fiber were observed in the experimental liquor.

\section{CONCLUSION}

A sodium silicate $\left(\mathrm{Na}_{2} \mathrm{O} 12.92 \%, \mathrm{SiO}_{2}, 15.87 \%\right.$, modulus 1.27) was prepared with a silicon tetrachloride from a polysilicon by-product. Then, the sodium silicate was composited with hydrazine and protease to get a new swelling agent $(67 \%$ sodium silicate, $17 \%$ hydrazine, $3 \%$ protease). The compound showed a better swelling effect with comparable void spaces in fiber bundles. Also, there was less total solid in the swelling effluents, showing this swelling process with the compound is a cleaner process.

\section{Acknowledgement}

The authors wish to thank Doctoral Scientific Fund Project of the Ministry of Education (Item No. 20130181130009) for finance support.
Aşa cum arată Tabelul 5, valoarea solidelor totale ale probelor experimentale a fost mai mică $(1,0547 \mathrm{~g} / 100 \mathrm{ml})$ şi a scăzut cu $33 \%$ comparativ cu proba martor. De obicei, varul are o contribuţie majoră la conţinutul de TS din efluentul de la cenuşărire din cauza solubilităţii sale mai mici. Din moment ce agentul de gonflare SSC nu conţine var, s-au eliminat TS provenite de la var din efluentul rezultat. S-a observat doar prezenţa părului şi a unor bucăţi mici de fibre în efluentul rezultat din procesul experimental.

\section{CONCLUZII}

S-a preparat un silicat de sodiu $\left(\mathrm{Na}_{2} \mathrm{O} 12,92 \%\right.$, $\mathrm{SiO}_{2}, 15,87 \%$, modulul 1,27) utilizând tetraclorură de siliciu dintr-un produs secundar al siliciului policristalin. Silicatul de sodiu a fost apoi amestecat cu hidrazină şi protează pentru a obţine un nou agent de gonflare (67\% silicat de sodiu, 17\% hidrazină, 3\% protează). Compusul a prezentat un efect de gonflare mai bun, $\mathrm{cu}$ un nivel comparabil al spaţiilor goale dintre fasciculele de fibre. De asemenea, au existat mai puţine materii solide totale în efluenţii de la gonflare, indicând că acest proces de gonflare care utilizează compusul dezvoltat este un proces mai curat.

\section{Mulţumiri}

Autorii doresc să mulţumească Ministerului Educaţiei pentru sprijinul financiar acordat prin intermediul Proiectului de Finanţare a Cercetării Ştiinţifice în cadrul Doctoratelor (nr. 20130181130009). 


\section{REFERENCES}

1. Thanikaivelan, P., Rao, J.R., Nair, B.U., Ramasami, T., J. Trends Biotechnol., 2004, 22, 4, 181-188.

2. Huber, C.F., Satyendra, M.D., J. Am. LeatherChem. Assoc., 1990, 85, 8, 276-286.

3. Albu, L., Popescu, M., Deselnicu, V., Albu, E., Zainescu, G., Revista de Pielarie Incaltaminte (Leather and Footwear Journal), 2011, 11, 3, 211-220.

4. Saravanabhavan, S., Aravindhan, R., Thanikaivelan, P., Chandrasekaran, B., Rao, J.R, Nair, B.U., J. Soc. Leather Technol. Chem., 2003, 87, 4, 149-158.

5. Thanikaivelan, P., Rao, J.R., Nair, B.U., J. Soc. Leather Technol. Chem., 2000, 84, 6, 276-284.

6. Saravanabhavan, S., Thanikaivelan, P., Rao, J.R., Nair, B.U., J. Environ. Sci. Technol., 2005, 39, 10, 3776-3783.

7. Saravanabhavan, S., Thanikaivelan, P., Rao, J.R., Nair, B.U., Ramasami, T., J. Environ. Sci. Technol., 2008, 42, 5, 17311739.

8. Saravanabhavan, S., Thanikaivelan, P., Rao, J.R., Nair, B.U., J. Environ. Sci. Technol., 2005, 39, 10, 3776-3783.

9. GB/T 4209-2008, Sodium Metasilicate, Chinese Standard.

10. Mantle, M., Allen, A.A., Biochem. Soc. Trans., 1978, 6, 3, 607-9.

11. Albu, L., Zainescu, G., Voicu, P., Ding, Z.W., Pang, X.Y., Revista de Pielarie Incaltaminte (Leather and Footwear Journal), 2012, 12, 4, 271-284.

Article received/Data primirii articolului: 03.04.2015

Accepted/Acceptat la data: 11.06.2015 